\title{
CONVEXITY AND UNIQUENESS IN AN INVERSE PROBLEM OF POTENTIAL THEORY
}

\author{
HENRIK SHAHGHOLIAN
}

(Communicated by J. Marshall Ash)

\begin{abstract}
Let $\Omega_{1}$ and $\Omega_{2}$ be two bounded domains in $\mathbb{R}^{n}$ whose intersection is convex. Suppose moreover that their volume potentials coincide in the complement of their union. Then $\Omega_{1}=\Omega_{2}$.
\end{abstract}

In 1938, Novikov [4] proved that if two bounded, convex (or even starshaped) domains have the same volume potential in the complement of their union, then they are identical. In [5] Zalcman asked whether this is true if just one of the domains is assumed to be convex. Also, cf. [3, p. 86]. In our efforts to investigate this problem we were able to prove the following: If the intersection of two bounded domains, which have the same volume potential in the complement of their union, is convex, then they are identical.

Let us introduce some notation. For a bounded domain $D$ we define $U^{D}$ to be the volume potential of $D$, i.e.,

$$
U^{D}(x)=c_{n} \int_{D} \frac{d y}{|x-y|^{n-2}}, \quad x \in \mathbb{R}^{n},
$$

where $c_{n}$ is a normalization factor so that $\Delta U^{D}=1$ in $D$. We also define the modified Schwarz potential (MSP) of a bounded domain $D$ with respect to $\mu$ to be the solution of $\Delta u=\chi_{D}-\mu$ (in the sense of distribution) and $u=0$ on $\mathbb{R}^{n} \backslash D$, where $\mu$ is a distribution supported in $\bar{D}$ and $\chi_{D}$ is the characteristic function of $D$.

Theorem 1. Let $\Omega_{1}$ and $\Omega_{2}$ be two bounded domains in $\mathbb{R}^{n}$, whose intersection $\Omega_{1} \cap \Omega_{2}$ is convex. Suppose moreover $U^{\Omega_{1}}=U^{\Omega_{2}}$ in $\mathbb{R}^{n} \backslash\left(\Omega_{1} \cup \Omega_{2}\right)$. Then $\Omega_{1}=\Omega_{2}$.

Remark 1. In Theorem 1 we see, by putting $|x|^{n-2} U^{\Omega_{1}}=|x|^{n-2} U^{\Omega_{2}}$ and letting $|x| \rightarrow \infty$, that $\Omega_{1}$ and $\Omega_{2}$ have the same volume, therefore, $\Omega_{1} \backslash \Omega_{2}$ and $\Omega_{2} \backslash \Omega_{1}$ are nonempty unless they are identical. It also follows that $\Omega_{1} \cap \Omega_{2}$ is not empty, else $U^{\Omega_{1}}$ can be continued harmonicly into $\mathbb{R}^{n}$ and violate Liouville's theorem.

The proof of Theorem 1 is based on the following lemmas.

Received by the editors April 25, 1991.

1991 Mathematics Subject Classification. Primary 31B20.

Key words and phrases. Convexity, volume potential, inverse problem. 
Lemma 2 (Gustafsson). In Theorem 1 , let $\Omega_{1} \not \equiv \Omega_{2}$. Then each domain $\Omega_{j}$ $(j=1,2)$ admits a MSP $u_{j}$ that is $C\left(\mathbb{R}^{n}\right)$, zero outside $\Omega_{j}$, and satisfies $\Delta u_{j}=\chi_{\Omega_{j}}-\mu$ where $\mu$ is a distribution supported in $\bar{\Omega}_{1} \cap \bar{\Omega}_{2}$.

Proof. Define

$$
U= \begin{cases}U^{\Omega_{1}} & \text { in } \mathbb{R}^{n} \backslash \Omega_{1}, \\ U^{\Omega_{2}} & \text { in } \mathbb{R}^{n} \backslash \Omega_{2} .\end{cases}
$$

As $U^{\Omega_{1}}=U^{\Omega_{2}}$ in $\mathbb{R}^{n} \backslash\left(\Omega_{1} \cup \Omega_{2}\right)$ this definition is consistent. Now extend $U$ to $\mathbb{R}^{n}$ as a continuous function and set $\mu=\Delta U$. Then $\mu$ is a distribution supported in $\bar{\Omega}_{1} \cap \bar{\Omega}_{2}$. Set $u_{j}=U^{\Omega_{j}}-U$ for $j=1,2$. Then $u_{j}$ satisfies the conditions stated in the lemma.

Remark 2. In Lemma 2 we can even assume the function $u_{1}\left(u_{2}\right)$ to be $C^{1}$ in a neighbourhood of any point $y \in \partial \Omega_{2} \cap \Omega_{1} \quad\left(\partial \Omega_{1} \cap \Omega_{2}\right)$. This will be needed for Lemma 4, where we will use the fact that the derivative of $u_{1}\left(u_{2}\right)$ at these points exists.

The following lemma is a modification of a result due to Caffarelli [1, Lemma 1].

Lemma 3. For $\Omega_{j}(j=1,2)$ in Theorem $1, \Omega_{1} \not \equiv \Omega_{2}$, and $u_{j}$ in Lemma 2 the following hold:

$$
\sup _{\partial \Omega_{2}} u_{1} \geq d_{1}^{2} / 2, \quad \sup _{\partial \Omega_{1}} u_{2} \geq d_{2}^{2} / 2,
$$

where $d_{1}=\sup _{x \in \Omega_{1}} \operatorname{dist}\left(x, \Omega_{1} \cap \Omega_{2}\right)$ and $d_{2}=\sup _{x \in \Omega_{2}} \operatorname{dist}\left(x, \Omega_{1} \cap \Omega_{2}\right)$.

Proof. Let $y \in \partial\left(\Omega_{1} \cap \Omega_{2}\right)$ and $z \in \partial \Omega_{1}$ be two points such that $d_{1}=|z-y|$. We may, by rotation and translation, assume $y$ is the origin and $z=\left(z_{1}, 0^{\prime}\right)$. Now set $\Omega_{1}^{\prime}=\left\{x \in \Omega_{1}: x_{1}>0\right\}$ and let $\left\{z^{j}\right\}$ be a sequence in $\Omega_{1}^{\prime}$ converging to $z$ and satisfying $u_{1}\left(z^{j}\right) \geq 0$ (as $\Delta u_{1} \geq 0$ near $z$ the existence of such points is obvious). Define now

$$
w(x)=u_{1}(x)-u_{1}\left(z^{j}\right)-\frac{1}{2}\left(x_{1}-z_{1}^{j}\right)^{2} \quad \text { in } \Omega_{1}^{\prime},
$$

and observe that $w\left(z^{j}\right)=0$. Then $w$, being harmonic in $\Omega_{1}^{\prime}$, attains its positive maximum on the boundary of $\Omega_{1}^{\prime}$. Since $w<0$ on $\partial \Omega_{1}$, the maximum value is attained at $x_{1}=0$ and it is positive. Now letting $z^{j} \rightarrow z$ we obtain

$$
\sup _{\partial \Omega_{2}} u_{1} \geq \sup _{x_{1}=0} u_{1} \geq z_{1}^{2} / 2=d_{1}^{2} / 2,
$$

where the first inequality is a consequence of the maximum principle, applied to $u_{1}$ in $\Omega_{1} \backslash \Omega_{2}$. One, similarly, proves the second statement. Thus the lemma is proved.

Lemma 4. Let $\Omega_{1}$ and $\Omega_{2}$ be as in Theorem $1, \Omega_{1} \not \equiv \Omega_{2}$ and $u_{1}, u_{2}$ as in Lemma 2. Then at least one of the following holds:

(1) There is a point $y^{1} \in \Omega_{1} \backslash \Omega_{2}$ such that $d_{1}^{2} / 2<-u_{1}\left(y^{1}\right)$ and $\left|\nabla u_{1}\right|\left(y^{1}\right)$ $=0$.

(2) There is a point $y^{2} \in \Omega_{2} \backslash \Omega_{1}$ such that $d_{2}^{2} / 2<-u_{2}\left(y^{2}\right)$ and $\left|\nabla u_{2}\right|\left(y^{2}\right)$ $=0$. 
Proof. Let $u_{j}(j=1,2)$ be the MSP of $\Omega_{j}$ with respect to $\mu$ (see Lemma 2). Set

$$
W_{1}=\left\{x \in \Omega_{1} \backslash \Omega_{2}: u_{1}(x)<0\right\}, \quad W_{2}=\left\{x \in \Omega_{2} \backslash \Omega_{1}: u_{2}(x)<0\right\},
$$

and define $v=\left(u_{1}-u_{2}\right)^{2}$. Then $v$ is subharmonic in $\mathbb{R}^{n} \backslash\left(\overline{W_{1} \cup W_{2}}\right)$ and it tends to zero at infinity. Hence, by the maximum principle, $v$ attains its maximum value at $y^{1} \in \partial W_{1} \cup \partial W_{2}$. If $W_{1} \cup W_{2}$ is empty then $u_{1}=u_{2}$, which implies $\Omega_{1}=\Omega_{2}$, and the proof will be completed. So assume it is not empty. Then as $u_{1}=u_{2}=0$ on $\left(\partial W_{1} \cup \partial W_{2}\right) \backslash\left(\partial \Omega_{1} \cup \partial \Omega_{2}\right)$, we conclude that $y^{1} \in\left(\partial \Omega_{1} \cup \partial \Omega_{2}\right) \cap\left(\partial W_{1} \cup \partial W_{2}\right)$, i.e., $y^{1} \in\left(\partial \Omega_{1} \cap \partial W_{2}\right) \cup\left(\partial \Omega_{2} \cap \partial W_{1}\right)$. Without loss of generality, let $y^{1} \in \partial \Omega_{2} \cap \partial W_{1}$. Then

$$
-u_{1}\left(y^{1}\right)=\sup _{\partial \Omega_{2}} \sqrt{v}>\sup _{\partial \Omega_{2}} u_{1} \geq d_{1}^{2} / 2
$$

the last inequality follows from Lemma 3. By Remark 2 we may assume $v$ is $C^{1}$ at $y^{1}$. Now either $|\nabla v|\left(y^{1}\right)=0$, which (since $u_{2}$ has vanishing Cauchy-data) will complete the proof, or $\nu . \nabla v\left(y^{1}\right)>0$ for some vector $\nu$ pointing outward $\Omega_{2}$. Since $u_{2}$ has vanishing Cauchy-data at $y^{1}$, we obtain $\nu . \nabla u_{1}^{2}\left(y^{1}\right)>0$. As $u_{1}\left(y^{1}\right)<0$ we arrive at $\nu . \nabla u_{1}\left(y^{1}\right)<0$. Thus $u_{1}$ decreases strictly in direction $\nu$. Therefore there is another point in $\Omega_{1} \backslash \bar{\Omega}_{2}$, which we (again) label $y^{1}$, at which $u_{1}$ attains its minimum value and consequently $\left|\nabla u_{1}\right|\left(y^{1}\right)=0$. This completes the proof.

Proof of Theorem 1. Suppose the conclusion in the theorem does not hold. Then, by Lemmas 2 and 4, we may assume that the MSP $u_{1}$ of $\Omega_{1}$ exists and satisfies:

(1) $\Delta u_{1}=1$ in $\Omega_{1} \backslash \Omega_{2}$ (which, by Remark 1, is not empty),

(2) there is a point $y^{1} \in \Omega_{1} \backslash \Omega_{2}$ such that $d_{1}^{2} / 2<-u_{1}\left(y^{1}\right)$ and $\left|\nabla u_{1}\right|\left(y^{1}\right)$ $=0$.

We may obviously assume that $u_{1}\left(y^{1}\right)=\inf _{\Omega_{1} \backslash \Omega_{2}} u_{1}$. Now by rotation and translation we may assume $y^{1}$ is the origin and $\Omega_{1} \cap \Omega_{2}$ is in the half-space $\left\{x: x_{1}<0\right\}$. Set $v=\frac{1}{2} x_{1}^{2}-u_{1}$ in $\Omega_{1}^{\prime}=\left\{x \in \Omega_{1}: x_{1}>0\right\}$. Then $v$, being harmonic in $\Omega_{1}^{\prime}$, attains its maximum value on $\Omega_{1}^{\prime}$ at the boundary $\partial \Omega_{1}^{\prime}$. By (2) and $u_{1}=0$ on $\partial \Omega_{1}$ we conclude that this maximum value is attained on $\partial \Omega_{1}^{\prime} \backslash \partial \Omega_{1}$, hence at the origin. Therefore, by Hopf's maximum principle [2],

$$
0>\frac{\partial v}{\partial x_{1}}(0)=0
$$

since both $\frac{1}{2} x_{1}$ and $u_{1}$ have vanishing gradient at the origin. Thus a contradiction is obtained and the theorem is proved.

\section{REFERENCES}

1. L. A. Caffarelli, Compactness methods in free boundary problems, Partial Differential Equations 5 (1980), 427-448.

2. E. Hopf, A remark on linear elliptic differential equations of second order, Proc. Amer. Math. Soc. 3 (1952), 791-793.

3. V. Isakov, Inverse source problems, Math. Surveys Monographs, vol. 34, Amer. Math. Soc., Providence, RI , 1990. 
4. P. S. Novikov, Sur le probème inverse du potentiel, Dokl. Akad. Nauk SSSR 18 (1938), 165-168.

5. L. Zalcman, Some inverse problems of potential theory, Contemp. Math., vol. 63, Amer. Math. Soc., Providence, RI, 1987, pp. 337-350.

Department of Mathematics, Royal Institute of Technology, S-100 44 Stockholm 70 , SWEDEN

E-mail address: henriks@math.kth.se 